LA-UR- $08-7871$

Approved for public release;

distribution is unlimited.

Title: Energy Release and Transfer in Solar Flares:

Simulations of Three-dimensional Reconnnection

Author(s): Joachim Birn, LANL/ISR-1

L. Fletcher, University of Glasgow, Scotland

Michael Hesse, NASA Goddard Space Flight Center

T. Neukirch, University of St. Andrews, Scotland

Intended for: Astrophysical Journal

EST. 1943

Los Alamos National Laboratory, an affirmative action/equal opportunity employer, is operated by the Los Alamos National Security, LLC for the National Nuclear Security Administration of the U.S. Department of Energy under contract DE-AC52-06NA25396. By acceptance of this article, the publisher recognizes that the U.S. Government retains a nonexclusive, royalty-free license to publish or reproduce the published form of this contribution, or to allow others to do so, for U.S. Government purposes. Los Alamos National Laboratory requests that the publisher identify this article as work performed under the auspices of the U.S. Department of Energy. Los Alamos National Laboratory strongly supports academic freedom and a researcher's right to publish; as an institution, however, the Laboratory does not endorse the viewpoint of a publication or guarantee its technical correctness. 


\title{
Energy Release and Transfer in Solar Flares: Simulations of Three-dimensional Reconnection
}

\author{
J. Birn ${ }^{1}$, L. Fletcher ${ }^{2}$, M. Hesse ${ }^{3}$ and T. Neukirch ${ }^{4}$ \\ ${ }^{1}$ Los Alamos National Laboratory, Los Alamos, NM 87545 \\ ${ }^{2}$ University of Glasgow, Scotland \\ ${ }^{3}$ NASA Goddard Space Flight Center, Greenbelt, MD 20771 \\ ${ }^{4}$ University of St. Andrews, Scotland
}

jbirn@lanl.gov

\begin{abstract}
Using three-dimensional magnetohydrodynamic (MHD) simulations we investigate energy release and transfer in a three-dimensional extension of the standard two-ribbon flare picture. In this scenario reconnection is initiated in a thin current sheet (suggested to form below a departing coronal mass ejection) above a bipolar magnetic field. Two cases are contrasted: an initially force-free current sheet (low beta) and a finite-pressure current sheet (high beta), where beta represents the ratio between gas (plasma) and magnetic pressure. The energy conversion process from reconnection consists of incoming Poynting flux turned into up- and downgoing Poynting flux, enthalpy flux and bulk kinetic energy flux. In the low-beta case, the outgoing Poynting flux is the dominant contribution, whereas the outgoing enthalpy flux dominates in the high-beta case. The bulk kinetic energy flux is only a minor contribution in the downward direction. The dominance of the downgoing Poynting flux in the low-beta case is consistent with an alternative to the thick target electron beam model for solar flare energy transport, suggested recently by Fletcher and Hudson (2008), whereas the enthalpy flux may act as an alternative transport mechanism. For plausible characteristic parameters of the reconnecting field configuration, we obtain energy release time scales and and energy output rates that compare favorably with those inferred from observations for the impulsive phase of flares. Significant enthalpy flux and heating are found even in the initially force-free case with very small background beta, resulting mostly from adiabatic compression rather than Ohmic dissipation. The energy conversion mechanism is most easily understood as a two-step process (although the two steps may occur essentially simultaneously): The first step is
\end{abstract}


the acceleration of the plasma by Lorentz forces in layers akin to the slow shocks in the Petschek reconnection model, involving the conversion of magnetic energy to bulk kinetic energy. However, due to pressure gradient forces that oppose the Lorentz forces in approximate, or partial force balance, the accelerated plasma becomes slowed down and compressed, whereby the bulk kinetic energy is converted to heat, either locally deposited or transported away by enthalpy flux and deposited later. This mechanism is most relevant in the downflow region, which is more strongly governed by force balance; it is less important in the outflow above the reconnection site, where more energy remains in the form of fast bulk flow.

Subject headings: MHD-Sun: corona-Sun: magnetic fields

\section{Introduction}

There is little doubt that the energy released in solar flares stems from magnetic energy stored in the solar corona and that magnetic reconnection is the physical process enabling this release and its conversion into kinetic energy in the form of bulk flow energy, thermal and non-thermal particle energy. But the details of this energy transfer and conversion are still very poorly understood. Based on hard X-ray (HXR) luminosities, the total energy of the accelerated electron population with energies above $\sim 25 \mathrm{keV}$ is estimated at a few tens of percent of the total energy released in some flares. $\gamma$-ray luminosities give a similar energy fraction for ions, such that the total energy conversion to non-thermal particle kinetic energy may be as high as 50\% (Miller et al. 1997; Emslie et al. 2005). In the standard picture, the energization takes place in the corona, by a yet unidentified process, whereupon an electron beam is formed that hits the chromosphere and generates hard X-rays by bremsstrahlung. However it is not clear how the large number of accelerated particles inferred from this model can be produced over very short timescales, and from a relatively modest reservoir of coronal particles. We note here that the distinction between thermal and "accelerated" (or nonthermal) particles is based on the assumption that the thermal population has a Maxwellian distribution, while the non-Maxwellian, higher-energy, contributions can be distinguished, for instance, by their X-ray signatures in high-resolution HXR spectra from the Ramaty High Energy Solar Spectroscopic Imager (RHESSI). This distinction may be somewhat artificial when the bulk of the particle energy, or at least a major portion, is contained in the nonthermal population.

Observations indicate that the onset of a typical two-ribbon flare is usually preceded by the eruption of a filament and a coronal mass ejection (CME). In the most common view 
(e.g., Forbes and Acton 1996), the eruption results from a loss of equilibrium (catastrophe) or an MHD instability, such as a kink mode (e.g., Török et al. 2004; Török and Kliem 2005; Fan and Gibson 2007), initiated when a gradual change of the underlying configuration exceeds a critical threshold, or a magnetic buoyancy-driven instability (e.g., Manchester et al. 2004). The departing CME leaves behind a stretched magnetic field configuration with an embedded electric current sheet. The breakup of this current sheet by reconnection then leads to the energy release associated with the solar flare. In a slight modification of this picture, a current sheet forms below a rising filament and its disruption by reconnection releases energy to the CME/filament as well as to the flare below (Forbes and Priest 1995; Forbes 2000).

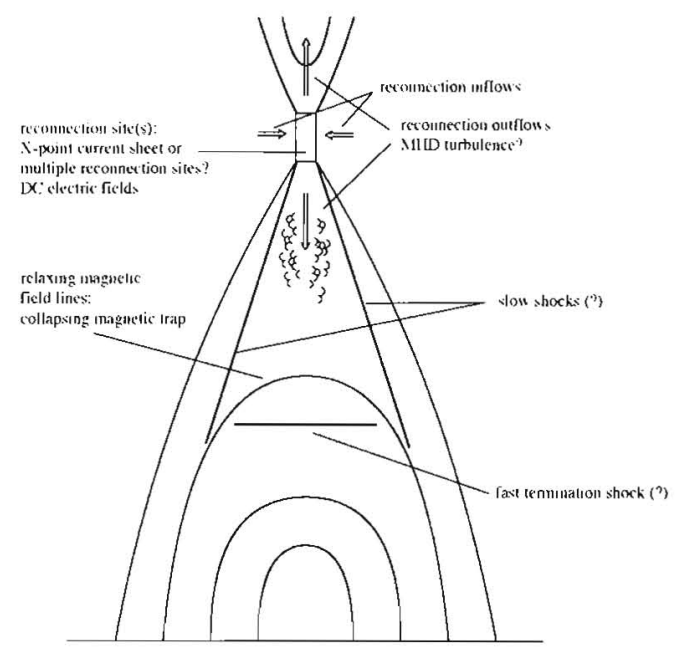

Fig. 1. - Sketch of a standard flare scenario with indication of possible acceleration sites (after Neukirch et al. 2007).

Fig. 1 illustrates the typical 2D flare scenario after the departure of the CME (after Neukirch et al. 2007), based on the so-called standard (CSHKP) model (Carmichael 1964; Sturrock 1966; Hirayama 1974; Kopp and Pneuman 1976). Specific acceleration mechanisms in this scenario include direct acceleration by the parallel electric field at the reconnection site(s) and secondary acceleration processes operating in the outflow region, such as turbulent heating, energization in the collapsing magnetic field (magnetic trap) below the reconnection site, and shock acceleration. The common feature of all of these models is that particle acceleration is located in the corona, which is attractive in that the coronal electron beam provides a single mechanism for both transporting energy to the solar chromosphere (where most of the flare radiant energy eventually emerges in the form of optical and ultraviolet emissions) and generating the HXR emission which is the electrons' primary diagnostic. However, it does come with the significant attendant challenges of the total electron number and rate 
required by the simple collisional thick target model or, alternatively, a viable model for the the geometry and stability of the often-invoked chromosphere-to-corona electron "resupply" mechanisms. Such considerations have been a challenge since the number implications of the coronal acceleration plus collisional thick target scenario were first deduced, and have if anything been thrown into sharper relief by the recent extremely high quality flare observations from RHESSI, the Transition Region and Coronal Explorer (TRACE) and ground-based optical and infrared instruments.

However, it is undeniable that the energy for a flare is stored in stressed coronal fields, apparently concentrated close to an active region polarity inversion line and relatively low in the corona (e.g. Schrijver 2007; Schrijver et al. 2008) and a large fraction of this energy must be transported to the chromosphere during the flare. Several authors have investigated MHD waves as a potential alternative heating or energy transport mechanism (e.g. Emslie and Sturrock 1982; Melrose 1992; Liu et al. 2006; Fletcher and Hudson 2008; Stasiewicz and Ekeberg 2008). In the case of a strong coronal magnetic field Fletcher and Hudson (2008) argued that the Poynting flux carried by Alfvénic wave pulses was a viable energy transport mechanism, also proposing some mechanisms for using this wave energy to accelerate electrons locally in the chromosphere where the energy is more readily dissipated than in the essentially collisionless corona. The agent for the redistribution of coronal magnetic free energy into the wave flux is magnetic reconnection, which permits the field to reconfigure. One of the significant theoretical questions surrounding such models is the distribution of the converted magnetic free energy between the various energy release channels available including the Poynting flux carried by waves. In an analytical treatment, Longcope and Priest (2007) demonstrated that the consequences of reconnection in a 2-D current sheet of finite extent is the generation of a fast magnetosonic wave pulse, carrying energy in the form of Poynting flux (accompanied by a small Ohmic heating component) away from the current sheet. As it travels, this pulse transforms magnetic into (bulk plasma) kinetic energy in the plasma distant from the current sheet.

It is the purpose of this paper to investigate the details of energy release, conversion and transport by reconnection within a three-dimensional (3D) extension of the flare scenario of Fig. 1. It has long been recognized that this scenario bears a strong resemblance to the substorm scenario in the Earth's magnetotail, which involves reconnection associated with the departure of a plasmoid (e.g., Kuperus 1976). Our modeling of reconnection therefore will draw some guidance from simulations of the initiation of reconnection and plasmoid release in the magnetotail. Specifically we will start from an already stretched arcadelike configuration, as expected below the departing CME, and include the formation of an intensified thin embedded current sheet from gradual boundary changes as a stage that leads into reconnection. This sequence is basically similar to that used by Birn et al. (2000). 
However, for a more realistic estimate of the energy partitioning, we consider significantly reduced background pressure with very low $\beta$ ( $\beta=$ ratio of plasma pressure to magnetic pressure), whereas the embedded current sheet itself may be of low or high $\beta$.

MHD simulations of reconnection in current sheets or arcade structures with application to solar flares have been conducted by various authors in two spatial dimensions (e.g., Forbes and Priest 1983; Forbes et al. 1989; Inhester et al. 1992; Yokoyama and Shibata 2001) and three dimensions (e.g., Kusano 2002; Ugai 2007). Our work goes beyond these particularly in using three-dimensional initial states, realistic low background pressure with low $\beta$ (as low as $2 \times 10^{-3}$ ), and by investigating details of the energy partitioning by the reconnection process.

In $\S 2$ we briefly discuss the chosen initial states and the numerical approach. In $\S 3$ we illustrate the dynamic evolution, followed by the detailed investigation of the energy flow and transfer in $\S 4$. Our investigation is based on one-fluid magnetohydrodynamic (MHD) simulations. Obviously, the one-fluid approximation does not permit a distinction between thermal and nonthermal populations or between ion and electron energization. The "heating" observed in the MHD simulations corresponds to an increase in energy density per unit mass, which need not be Maxwellian. In order to identify to what extent the energy partitioning found in the MHD simulations can be validated by particle approaches, we have also performed particle-in-cell (PIC) simulations, albeit of more localized reconnection problems. Details of these simulations will be reported in a future paper. Here we just note that they confirm our basic MHD results that only a minor amount of the energy released goes into bulk kinetic energy in the closed field line region or Joule dissipation.

\section{Initial States and Numerical Procedure}

Here and in the following we use dimensionless units, based on a characteristic magnetic field strength $B_{c}$, density $n_{c}$, and scale length $L_{c}$. For illustration and quantitative comparison with observed quantities, we will use

$$
\begin{aligned}
B_{c} & =100 \mathrm{G}=0.01 \mathrm{~T} \\
n_{c} & =2 \times 10^{15} \mathrm{~m}^{-3} \\
L_{c} & =10,000 \mathrm{~km}
\end{aligned}
$$

in the inflow region. These values lead to a velocity unit (Alfvén speed) $v_{c}=5000 \mathrm{~km} / \mathrm{s}$, time unit $t_{c}=L_{c} / v_{c}=2 \mathrm{~s}$, and energy unit $k T_{c}=B_{c}^{2} / \mu_{0} n_{c}=4 \times 10^{-14} \mathrm{~J}=250 \mathrm{keV}$. We use (mostly) standard international (SI) units with common notations with $k$ being the Boltzman constant. Our coordinate system is chosen such that $x$ is the coordinate 
perpendicular to the current sheet (horizontal axis in Fig. 1), $y$ is in the direction of the main current (perpendicular to the plane of Fig. 1), and $z$ is the vertical direction.

Our initial states are derived from the explicit three-dimensional equilibrium models given by Birn et al. (2003). These models are valid for configurations that vary most rapidly in one spatial direction (here, $x$ ). This is a reasonable approximation for the stretched field configuration below a departing CME. However, the field closer to the solar surface can be expected to be less stretched. Therefore we added a 3D dipole magnetic field with a location of the dipole below the photospheric surface. Since this superposition leads to fields that are no longer in exact force balance, we used a relaxation method (Hesse and Birn 1993) to obtain force balanced equilibria before starting the simulation of the dynamic evolution.

Two types of current sheet configurations are considered. One is initially force-free, that is, the current density vector is aligned with the magnetic field. In this type of configuration, the pressure of the magnetic field that reverses across the current sheet, $B_{z}$, is balanced by the magnetic pressure of the component in the perpendicular direction, $B_{y}$, in the direction of the peak current in the center of the sheet. This component is frequently called the "guide field" or shear field, as it introduces a shear of the magnetic field, corresponding to, and possibly caused by, a shear of foot points of field lines in the $y$ direction. In the other case, the guide field is assumed to be small or zero and the outside magnetic pressure is balanced by the plasma pressure inside the current sheet. A small uniform background plasma pressure of $1 \%$ of the magnetic pressure is added in all cases. The force-free and the non-force-free initial states are therefore also distinguished by low and high plasma $\beta$ in the current sheet, whereas the background has $\beta=0.01$ or even $\beta=0.002$. Such values appear reasonable above solar active regions (e.g., Gary 2001).

The evolution of the magnetic field is studied on the basis of a one-fluid MHD code. This code consists of an explicit, finite-difference, leapfrog scheme, based on two staggered meshes defined at alternate time steps (e.g., Birn et al. 1996, 2006). A nonlinear grid is used to increase the resolution in the regions of interest, such that about $1 / 2$ of the grid points lie within the current sheet. A quasi-viscous term, similar to flux-corrected transport algorithms (e.g., Book et al. 1975), damps oscillations on the grid scale, reduces the divergence between the quantities on the two meshes, and increases numerical stability. This algorithm is not used on the magnetic field to avoid introducing artificial diffusion and reconnection. As for the equilibrium, gravity is neglected. We further neglect radiation and heat conduction, assuming an adiabatic law with a ratio of specific heats $\gamma=5 / 3$ but including Ohmic heating. This assumption seems reasonable, as we focus on the energy release powering the impulsive phase of flares. We note that in our one-fluid code, mass density and pressure represent the sum of electron and ion (here simply proton) density and pressure. 


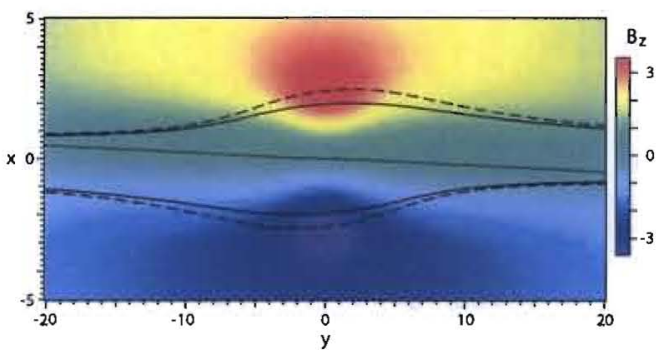

Fig. 2.- Normal magnetic field $B_{z}$ at the bottom boundary $z=0$ (color) after the completion of the converging foot point motion. The dotted line indicates $B_{z}=0$. The dashed and solid lines show the boundaries of field lines that close within the box before and after the foot point motion, respectively.

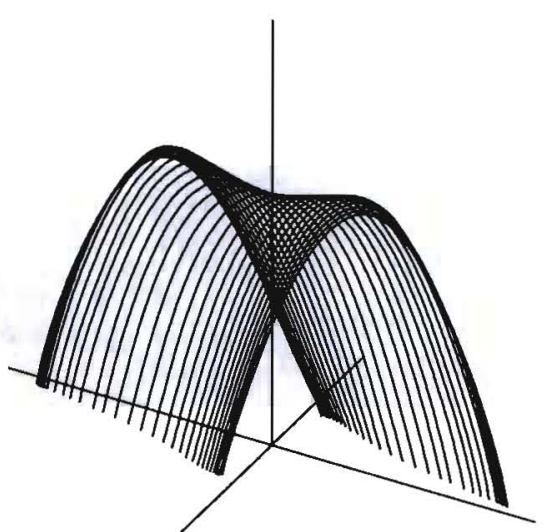

(a)

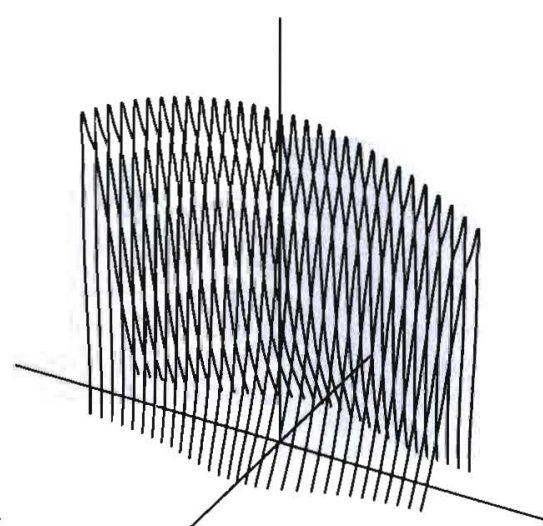

(b)

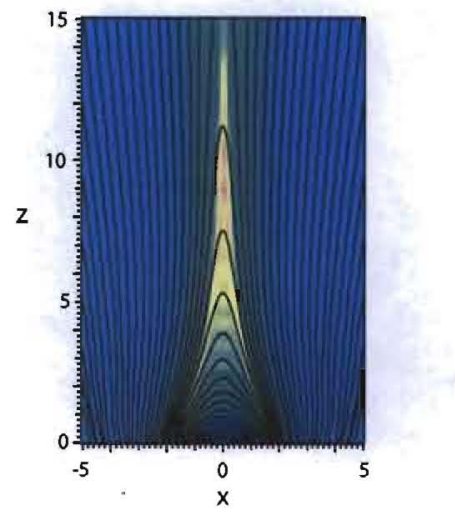

(c)

Fig. 3.- Perspective view of two characteristic magnetic field configurations after the completion of the converging foot point motion: (a) force-free, (b) shear-free initial state; (c) current density and magnetic field in the $x, z$ plane for the force-free case.

The boundary conditions consist of solid, ideally conducting walls at each of the boundaries $z=0, y= \pm y_{\max }= \pm 40$, and $x= \pm x_{\max }= \pm 10$, where all velocity components are set to zero (except for the initial phase, discussed below). Von Neumann boundary conditions $(\partial / \partial n=0)$ are imposed on density, pressure, and the tangential magnetic field components, except at the top boundary $z=60$, where an open outflow condition is assumed, such that $B_{x}$ and $B_{y}$ are convected with the plasma flow, while the normal magnetic field is held fixed. Line symmetry conditions are imposed around the $z$ axis, such that only the half box $x \geq 0$ needed to be simulated. For most runs, the number of grid cells in $x, y$, and $z$, are 64,80 , and 132, respectively, for the full box, using nonuniform grids that concentrate more points near the solar surface and the center of the current sheet. By varying the grid size and the time step we confirmed that the basic results reported here are not affected significantly be 
the spatial or temporal resolution.

As mentioned in the Introduction, our simulations include a slow phase of current intensification prior to the initiation of reconnection. In this phase we apply a slow converging motion at the bottom boundary $z=0$ in the $x$ direction towards the field reversal near $x=0$. This motion, with a maximum amplitude at $y=0$, is gradually turned on and off, as described in detail by Birn et al. (2000). During this phase, the resistivity is set to zero, allowing additional current to build or concentrate under the action of the slow driving.

Fig. 2 shows the boundary conditions (color-coded magnitude of $B_{z}$ at $z=0$ ) for the force-free initial state after the completion of this motion. The dotted line indicates the neutral line $B_{z}=0$. The dashed and solid lines show the boundaries of field lines that close within the box, before and after the foot point motion, respectively. The converging motion leads to an increase in the boundary field strength $B_{z}$ of about $70 \%$.

Fig. 3 gives a perspective view of magnetic field lines after the completion of this phase, (a) for the force-free initial state and (b) for a high- $\beta$ case without shear. In the latter case the current density increase can be inferred from the sharp bend in the field at the top of the field lines. However, the force-free configuration experiences a similar local increase in the current density, as shown in Fig. $3 \mathrm{c}$ in a $y=0$ cut. We should note that the force-free initial configuration does not remain exactly force-free, as a consequence of the motion. However, the plasma $\beta$ remains below $\sim 0.025$.

\section{Overview of the Dynamic Evolution}

To initiate the following, eruptive phase, we imposed a localized finite resistivity, centered near the peak of the current intensification, but kept fixed in time, given by

$$
\begin{aligned}
\eta & =\eta_{0} / \cosh s \\
s & =\left[\left(\frac{x}{L_{x}}\right)^{2}+\left(\frac{y}{L_{y}}\right)^{2}+\left(\frac{z-z_{0}}{L_{z}}\right)^{2}\right]^{1 / 2}
\end{aligned}
$$

with

$$
z_{0}=8, \quad L_{x}=2, \quad L_{y}=8, \quad L_{z}=4
$$

The maximum value $\eta_{0}=0.005$ of the resistivity, used in most of our simulations, corresponds to a Lundquist number (magnetic Reynolds number) of 200, based on the units of the initial state.

This resistivity, in combination with the current intensification from the previous phase 
leads to the onset of reconnection. The subsequent evolution of the magnetic field is illustrated by Fig. 4, (a) for the force-free initial state, and (b) for a shear-free initial state.

Here, $t=0$ marks the time when the resistivity is turned on. In the shear-free case the lines in Fig. 4b are the actual field lines, whereas the lines in Fig. 4a show the direction of the magnetic field in this plane but projections of the actual field lines deviate from these lines.
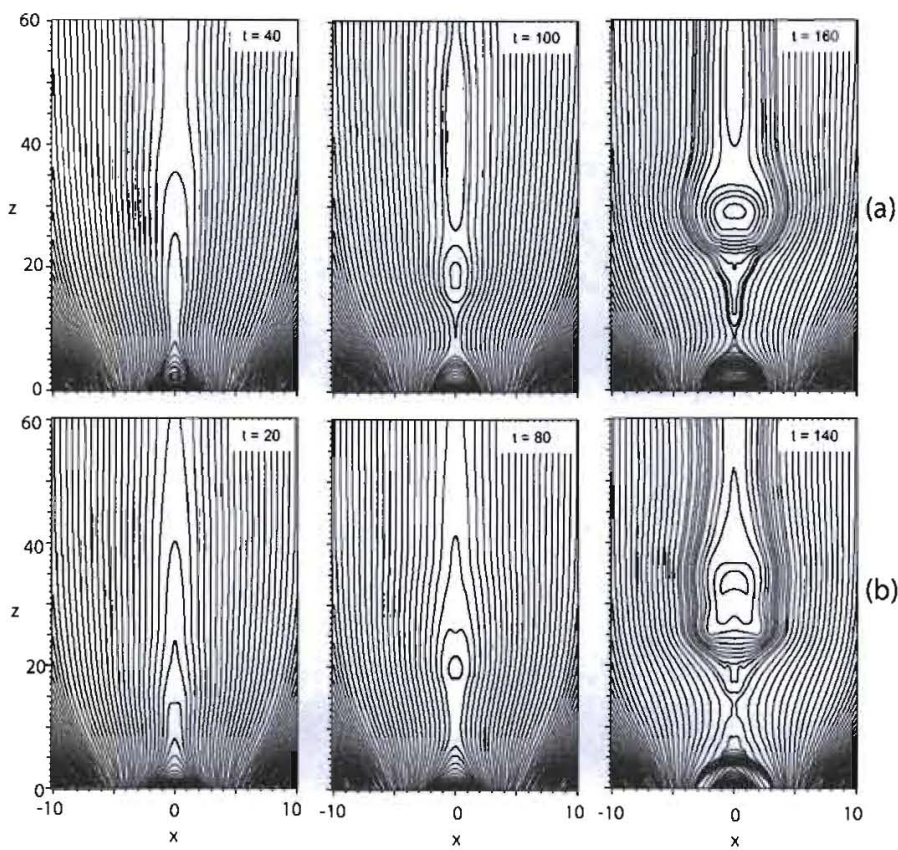

Fig. 4.- Evolution of the magnetic field in the $x, z$ plane for (a) the force-free and (b) a shear-free initial state.

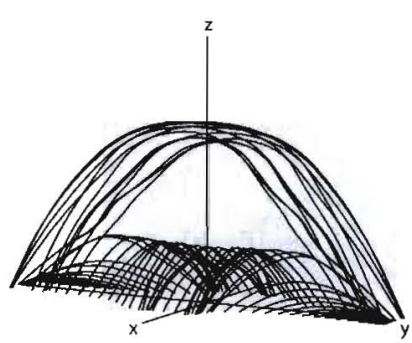

(a)

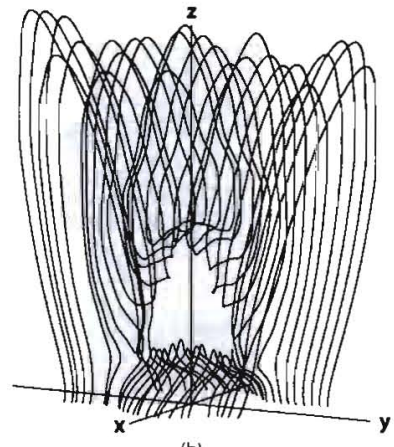

(b)

Fig. 5.-- Perspective view of magnetic field lines during plasmoid ejection, for (a) a force-free initial state and (b) a high- $\beta$ case with a very small shear field.

The magnetic field evolution is very similar for the two cases. However, the three- 
dimensional field structure differs significantly, as demonstrated by Fig. 5, which shows perspective views of actual field lines. Fig. 5 a again corresponds to a force-free initial state, while Fig. 5b represents a high- $\beta$ case with a small but finite shear field, showing a tightly wound flux rope, whereas the departing flux rope in Fig. 5a is only slightly twisted. In the latter case the flux rope field lines make only one half turn, whereas in Fig. 5b the flux rope field lines make about three turns. If the initial shear field is further reduced, the winding number increases. It is likely that the forces that would render such tightly wound flux ropes kink unstable in equilibrium contribute to the somewhat faster ejection observed in this case.

While the pressure for the force-free initial state changes only a little in the pre-onset phase, it changes drastically during reconnection, as demonstrated by Fig. 6 (6 top panels), in comparison to a shear-free case (3 bottom panels). There are two regions where a pressure increase can be seen in the top panels. One is the center of the current sheet right at the reconnection site. The reason for this pressure increase is the decrease of the guide field $B_{y}$ from the center of the current sheet outward in $x$, characteristic of the force-free initial state. As reconnection proceeds, the incoming field lines contain smaller and smaller guide fields. To maintain pressure balance in the $x$ direction the current sheet therefore contracts, increasing the pressure within, so that eventually the gas pressure contribution becomes comparable to, or even exceeds the magnetic pressure of the guide field.

The second region of pressure enhancement is the region where the downward collapsing reconnected magnetic flux tubes, which contain already preheated plasma, become shorter and further compressed. This process is also very dynamic; pressure enhancements can travel from the loop tops downward and back (due to our reflecting boundary). The propagation speed for this transport is the sound speed, which is initially small but becomes considerably enhanced also from the pressure and temperature enhancement.

The pressure increase shown in Fig. 6 results mainly from adiabatic compression. It is therefore also associated with a temperature (energy density) and density increase. That is demonstrated by Fig. 7, which shows the temperature in the $x, z$ plane for the initially forcefree case. Adopting our units (1), the maximum temperatures in the loops correspond to about $8 \mathrm{keV}$. We note again that this represents the sum of ion and electron energy densities. Assuming equal ion and electron temperatures, the maximum electron temperature would be $\sim 4 \mathrm{keV}$. This value is slightly higher (by a factor 3-10) than flare loop temperatures inferred from Yohkoh data (e.g., Feldman et al. 1995) or GOES data (e.g., Feldman et al. 1996), however comparable to the thermal temperatures obtained by fitting the flare hard X-ray spectrum. For example in the July 232002 event observed by RHESSI, Holman et al. (2003) found a temperature of around $3 \mathrm{keV}$. Note also that our units are somewhat arbitrary. The temperature values would decrease by a factor of 3 if the chosen background density 

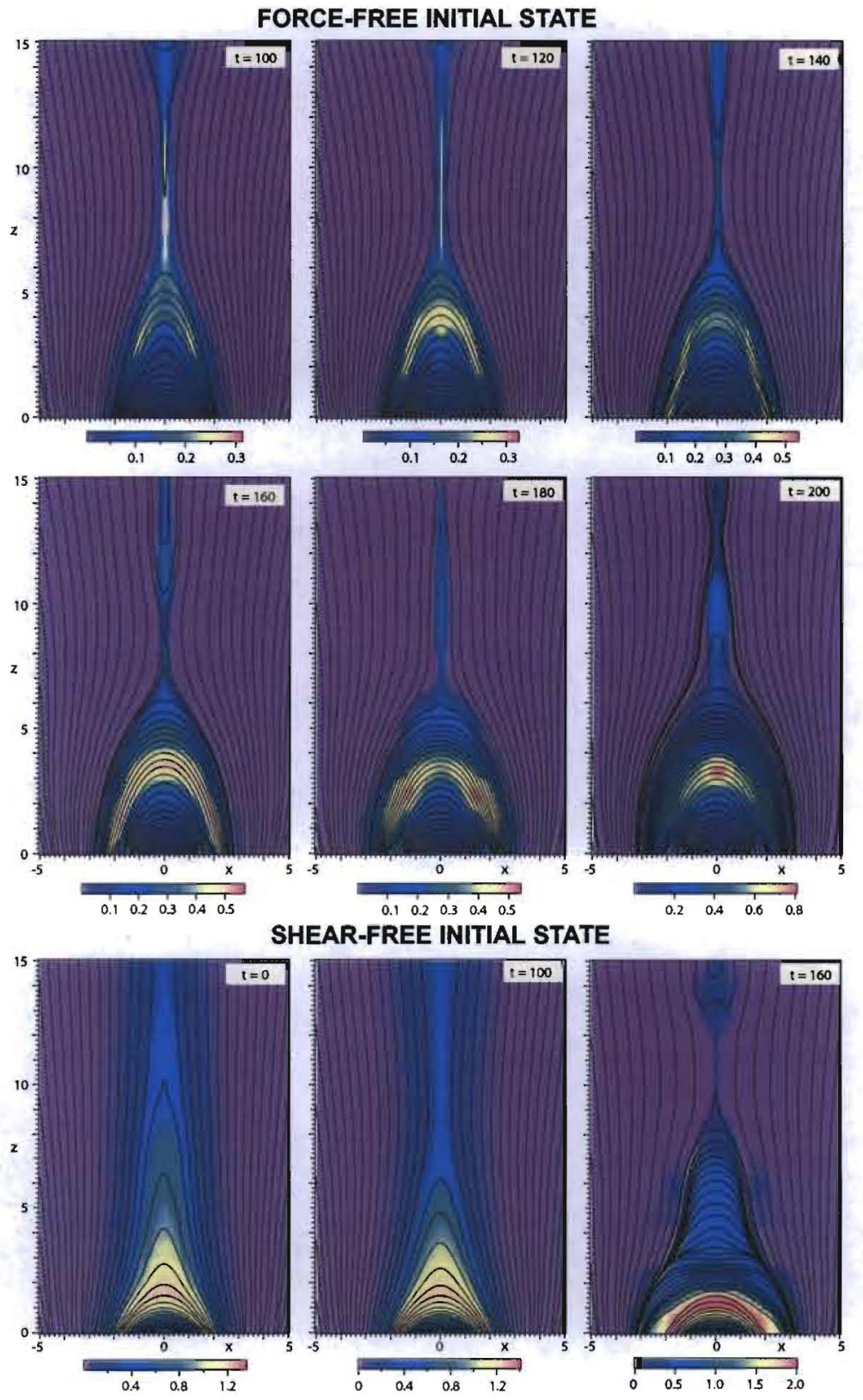

Fig. 6. - Evolution of the pressure (color-coded) and magnetic field in the $x, z$ plane for the force-free initial state ( 6 top panels) and a shear-free initial state ( 3 bottom panels). 

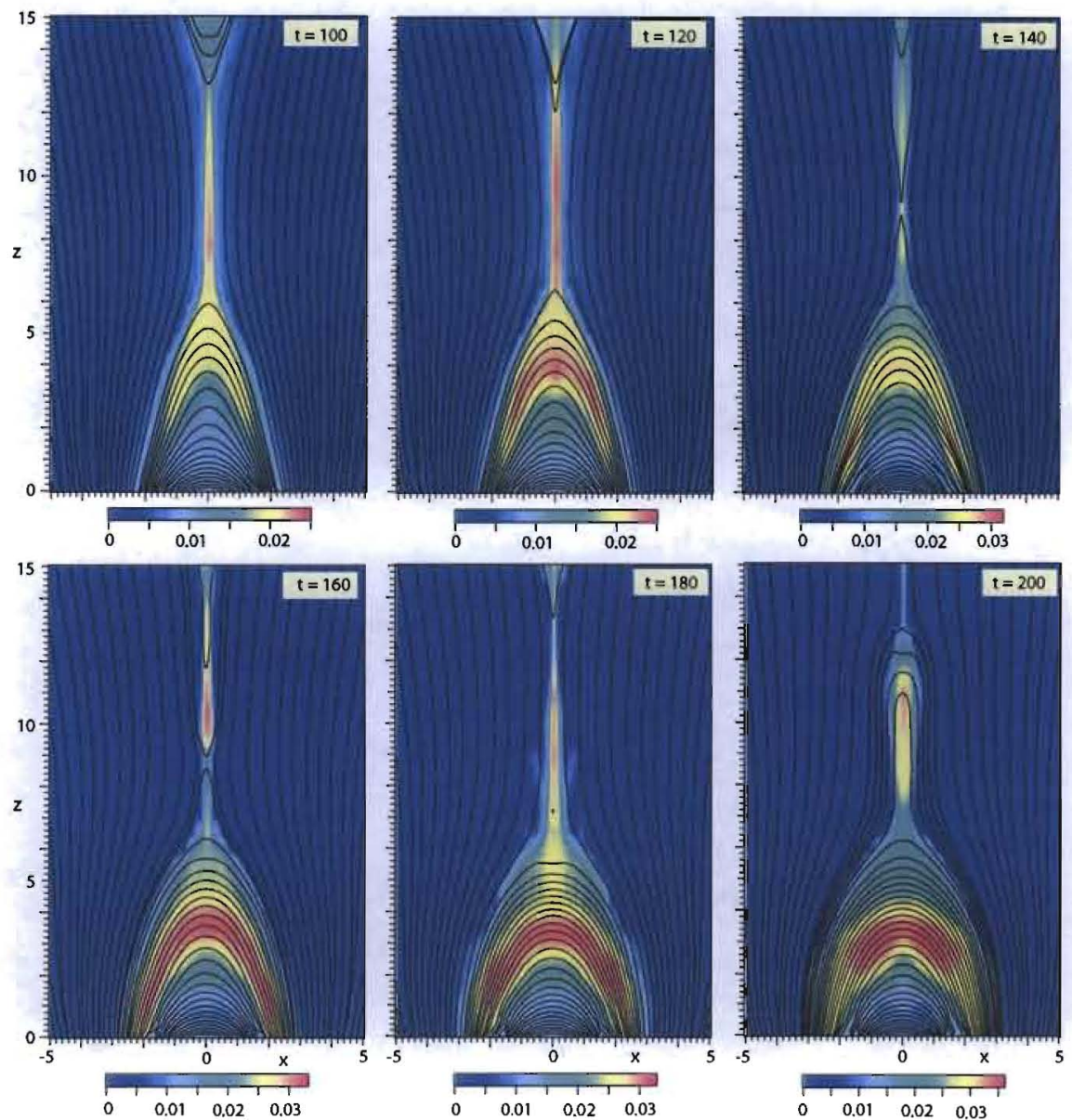

Fig. 7.- Evolution of the temperature (color-coded) and magnetic field in the $x, z$ plane for the force-free initial state. 
were higher by a factor of 3 . Furthermore our approach did not include major energy loss mechanisms, specifically, radiative losses, collisional losses and losses from transport through the lower boundary to the chromospheric plasma.

To study the effects of the background plasma $\beta$, we have run a simulation from a force-free initial state in which the background $\beta$ was reduced to 0.002 . This case, which is actually shown in Figure 7, led to almost identical results as the case with $\beta=0.01$, except for the lower background $\beta$. Thus the background temperature in Figure 7 corresponds to an electron temperature $T_{e} \approx 0.25 \mathrm{keV}$, assuming equal ion and electron temperatures. The peak temperatures, however, are not affected by the background $\beta$. The reason for this result will become clear in the following section, where we discuss the energy transfer mechanisms.

In contrast to the force-free case, in the shear-free, high- $\beta$ current sheet, the enhanced pressure already exists prior to reconnection and extends also along field lines downward (bottom panels of Figure 6). Therefore, pressure and temperature-enhanced loops are already present prior to reconnection, although the collapse of the reconnected flux tubes produces a further enhancement (last panel of Figure 6).

Figures 4, 6, and 7 show the field and plasma evolution in the standard 2D $x, z$ projection. However, the evolution is also characterized by significant variation in the perpendicular direction. To demonstrate this, Fig. 8 shows the vertical component of the plasma velocity $v_{z}$ for the force-free initial state at 3 successive times, together with magnetic field lines projected into the $y, z$ plane. Based on our chosen units in (1) the maximum speeds up and down correspond to $\sim 1000 \mathrm{~km} / \mathrm{s}$ (top) to $\sim 2500 \mathrm{~km} / \mathrm{s}$ (bottom). Figure 8 illustrates that the early nearly two-dimensional reconnection structure with flow away from a nearly straight separator line splits up into multiple reconnection sites. A possible cause may be the development of a ballooning or interchange type of mode, related to the development of finite pressure.

\section{Energy Transfer}

In this section we investigate the properties of energy conversion and transport associated with the reconnection process, focusing particularly on the conversion to energy downflow. In the one-fluid, MHD model there are three types of energy flux, the Poynting vector $\mathbf{S}$, describing electromagnetic energy flux

$$
\mathbf{S}=\mathbf{E} \times \mathbf{B} / \mu_{0}
$$




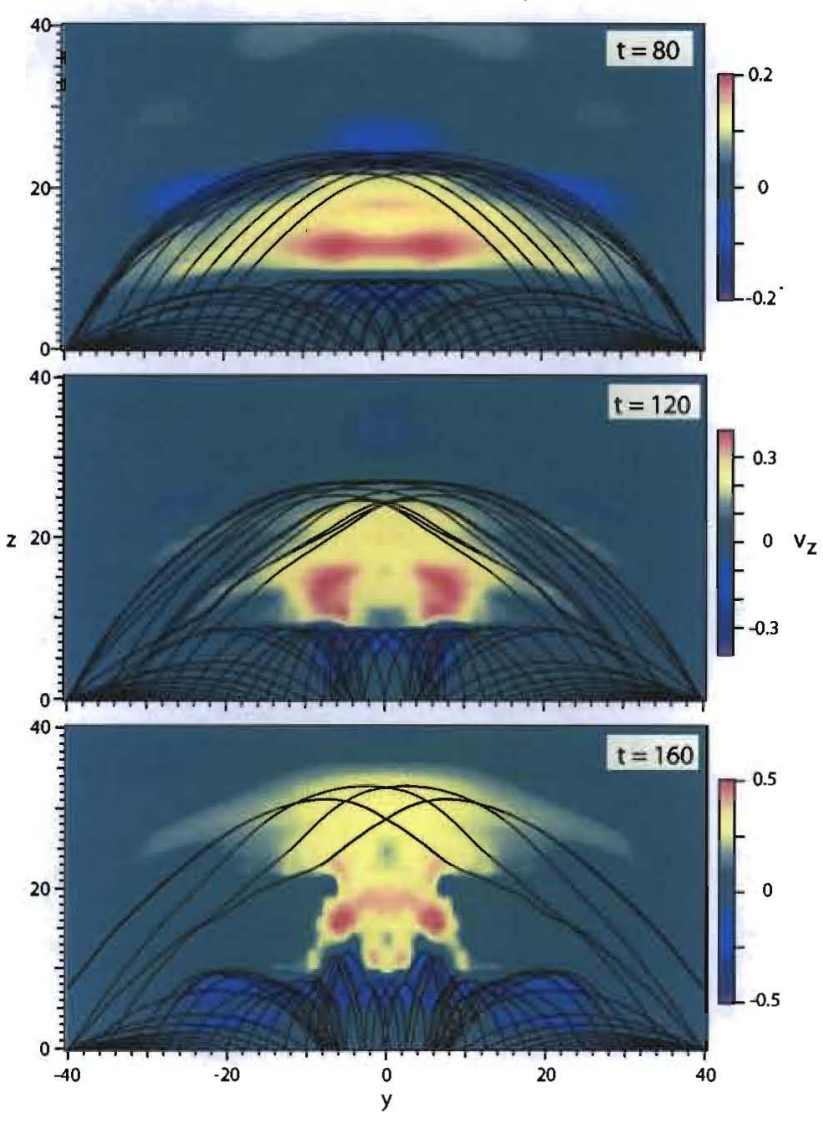

Fig. 8.- Vertical velocity component $v_{z}$ in the $y, z$ plane for the force-free initial case, indicating spatial and temporal variability of the reconnection site.

the enthalpy flux, describing the convective transport of thermal energy,

$$
\mathbf{H}=(u+p) \mathbf{v}
$$

where $u=p /(\gamma-1)$ is the internal (thermal) energy density and the contribution $p \mathbf{v}$ describes the effects of compressional work, and the bulk kinetic energy flux

$$
\mathbf{K}=\frac{1}{2} \rho v^{2} \mathbf{v}
$$

(Here we use dimensional units; using our dimensionless units is equivalent to setting $\mu_{0}=0$.)

These fluxes are governed by the following energy equations (e.g., Birn et al. 2005), consisting of the Poynting theorem,

$$
\frac{\partial}{\partial t} \frac{B^{2}}{2 \mu_{0}}=-\nabla \cdot\left(\frac{\mathbf{E} \times \mathbf{B}}{\mu_{0}}\right)-\mathbf{v} \cdot(\mathbf{j} \times \mathbf{B})-\eta j^{2}
$$




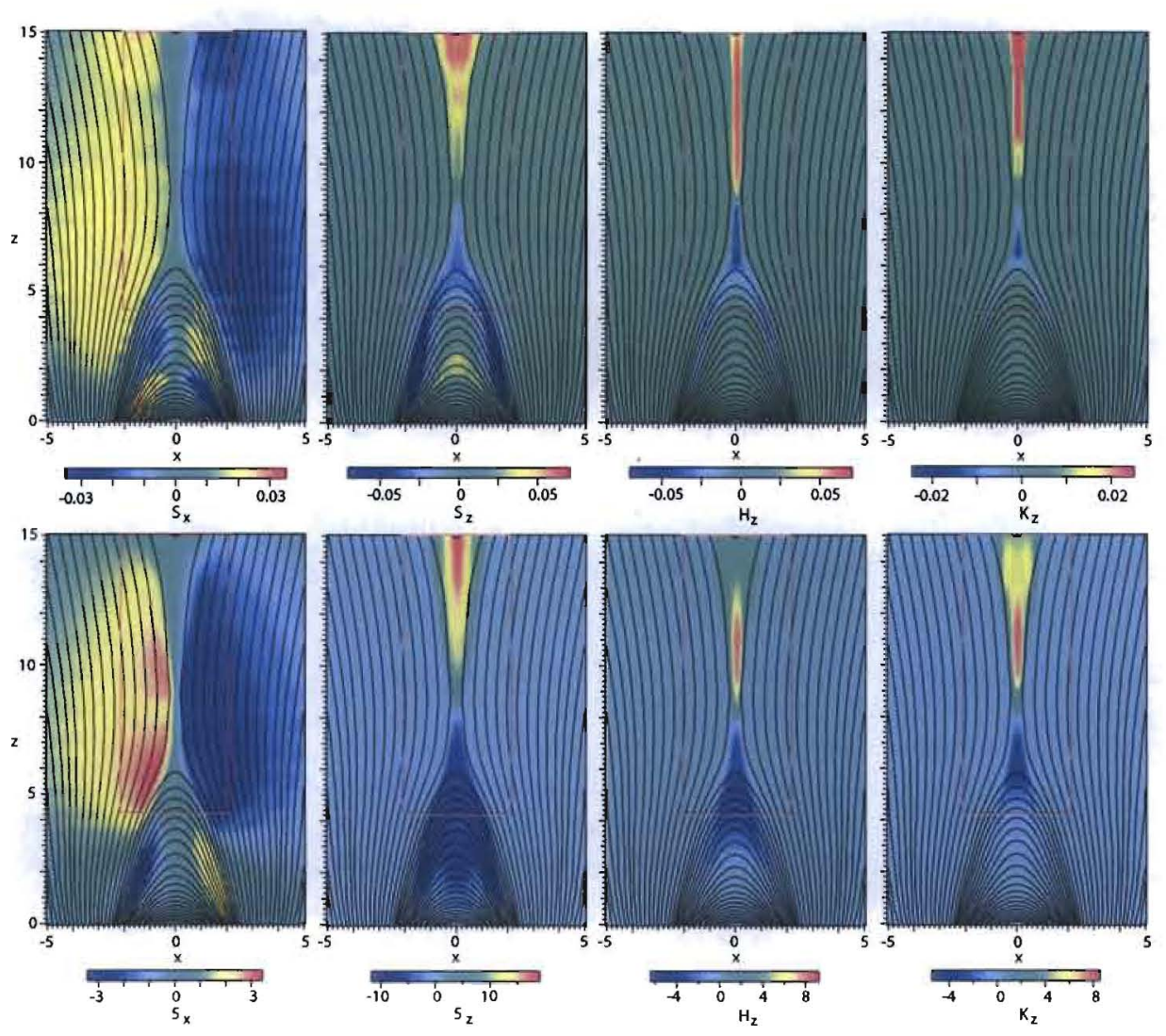

Fig. 9.- Energy transfer in the initially force-free case. The top row shows energy fluxes in the $x, z$ plane, the bottom row the fluxes integrated over $y$ : (from left to right) the Poynting vector components $S_{x}$ and $S_{z}$, the enthalpy flux component $H_{z}$, and the bulk kinetic energy flux component $K_{z}$. The solid black lines represent the magnetic field direction in the $x, z$ plane. The red rectangles indicate boxes used to integrate in- and outgoing energy fluxes shown in Figure 10.

an equation for the thermal energy,

$$
\frac{\partial u}{\partial t}=-\nabla \cdot[(u+p) \mathbf{v}]+\mathbf{v} \cdot \nabla p+\eta j^{2}
$$

and an equation governing bulk kinetic energy, which derives from mass and momentum conservation laws,

$$
\frac{\partial}{\partial t} \frac{\rho}{2} v^{2}=-\nabla \cdot\left(\frac{\rho}{2} v^{2} \mathbf{v}\right)+\mathbf{v} \cdot(\mathbf{j} \times \mathbf{B}-\nabla p)
$$

We note here again that in Equation (8) heat flux, i.e., thermal conduction, and radiative losses are neglected, and that the one-fluid description does not permit us to distinguish thermal and supra-thermal populations. 
Equations (7)-(9) describe both the conservation of energy and the transfer from one form of energy to another. For instance, magnetic energy can be transferred, via Ohmic dissipation, $\eta j^{2}$, to heat or, via acceleration by the Lorentz force, $\mathbf{j} \times \mathbf{B}$, to bulk kinetic energy. However, the Lorentz force may be balanced, in part or fully, by pressure gradient forces, which then also provides a transfer to thermal energy, via work done against the pressure gradient force, $\mathbf{v} \cdot \nabla p$.

Figure 9 illustrates the characteristic energy flow for the initially force-free case at the time of fastest reconnection, $t=120$, showing energy fluxes in the $x, z$ plane (top row) and integrated over $y$ (bottom row), together with the magnetic field in the $x, z$ plane (solid black lines): (from left to right) the Poynting vector components $S_{x}$ and $S_{z}$, the enthalpy flux component $H_{z}$, and the bulk kinetic energy flux component $K_{z}$, respectively. The figure clearly shows that incoming Pointing flux ( $S_{x}$, left panels) is converted to up and down going Poynting flux $\left(S_{z}\right.$, second panels from the left) and up and down going enthalpy flux $\left(H_{z}\right.$, third panels). Kinetic energy flux $\left(K_{z}\right.$, right panels) is significant only in the neighborhood of the reconnection site and in the upward direction. The positive and negative contributions to $S_{x}$ in the lower parts of the arcade structure (top row, second panel of Fig. 9) correspond to Alfvénic waves that arise particularly after the downgoing flux hits the bottom boundary and becomes reflected, as indicated by the blueish inner regions in the panel for $S_{z}$.

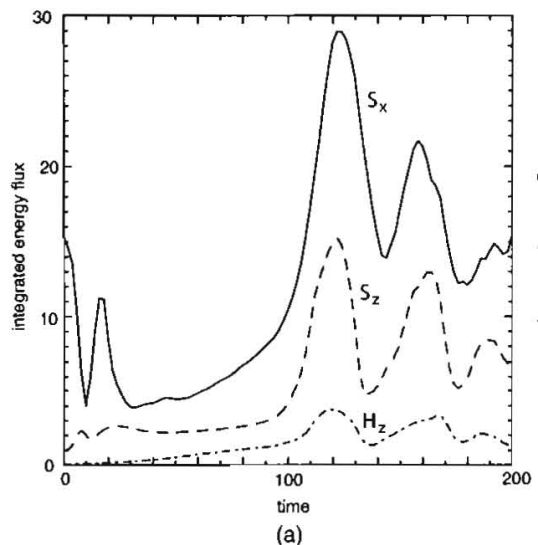

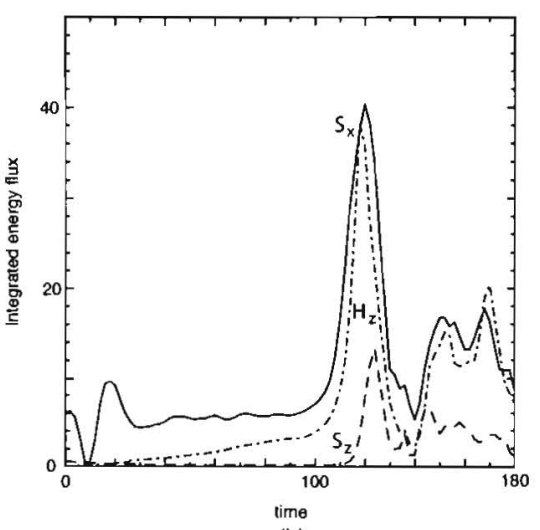

(b)

Fig. 10.- Energy fluxes into and down from the reconnection site as function of time, integrated over the boundaries of the red boxes indicated in Figure 9; (a) force-free initial state, (b) shear-free initial state. The solid lines show the Poynting flux $S_{x}$ into the reconnection site, the dashed lines the Poynting flux $S_{z}$ downward from the reconnection site, and the dash-dotted line the downward enthalpy flux $H_{z}$.

The temporal evolution of the energy release and transfer is shown in Figure 10, (a) for the force-free initial state, and (b) for a shear-free initial state. The solid lines show the 
Poynting flux $S_{x}$ into the reconnection site, the dashed lines the Poynting flux $S_{z}$ downward from the reconnection site, and the dash-dotted line the downward enthalpy flux $H_{z}$. These fluxes are integrated over the surfaces of a box $|x|<2,4<z<15,|y|<y_{\max }=40$, indicated by the red rectangles in Figure 9 . (The value of $y_{\max }$ is not critical because the main contributions stem from within $|y| \approx 10$.) The fluxes enter from the sides $|x|=2$, and go out through the top $z=15$ (not shown here) and bottom $z=4$. The incoming enthalpy and kinetic energy fluxes, as well as the downgoing kinetic energy flux, are very small and therefore not included. We further note that the in- and outgoing fluxes do not perfectly balance. This is primarily due to a decrease of magnetic energy within the box, which results from the fact that the incoming Poynting flux carries smaller guide field, and hence less magnetic energy than the outgoing Poynting flux.

It is instructive to apply our chosen dimensional units (1) to the fluxes in Figure 10. From a power unit of $4 \times 10^{22} \mathrm{~W}=4 \times 10^{29} \mathrm{erg} / \mathrm{s}$ we find peak downward energy fluxes of the order of $10^{24} \mathrm{~W}=10^{31} \mathrm{erg} / \mathrm{s}$ in bursts of $\sim 40-80 \mathrm{~s}$ duration. These numbers are quite commensurate with those for the impulsive phase of large flares.

Figure 10 demonstrates that the process of energy release and conversion to downgoing energy mainly consists of a redirection of Poynting flux and a conversion to enthalpy flux. In the (initially) force-free case the downward Poynting flux dominates over the enthalpy flux, whereas the opposite is true for the shear-free case with the high- $\beta$ current sheet. Note that even though the plasma $\beta$ in the force-free case was initially only 0.01 , a substantial enthalpy flux of about $20 \%$ of the Poynting flux is obtained in the downward fluxes. This relative amount does not change even if the background $\beta$ in our simulations is further reduced to 0.002. As we will see below, the reason for this result is the fact that the energy of the outgoing enthalpy flux does not stem from the simple compressional heating of the thermal inflow plasma but rather from a two-step process that involves acceleration by Lorentz forces and deceleration by pressure gradients, which may happen simultaneously in approximate force balance. Bulk kinetic energy flux is important only in the upward outflow region, which is not addressed here in detail.

To further clarify the transfer mechanisms, and to assess the possible role of our resistivity model, we have integrated the transfer terms in (7), (8) and (9) over a box $4.2<z<8.2$, $|x|<2.1$, and $|y|<y_{\max }=40$, covering the downflow region, defining

$$
\begin{aligned}
D_{J} & =\int \eta j^{2} d V \\
D_{L} & =\int \mathbf{v} \cdot(\mathbf{j} \times \mathbf{B}) d V \\
D_{P} & =\int \mathbf{v} \cdot \nabla p d V
\end{aligned}
$$




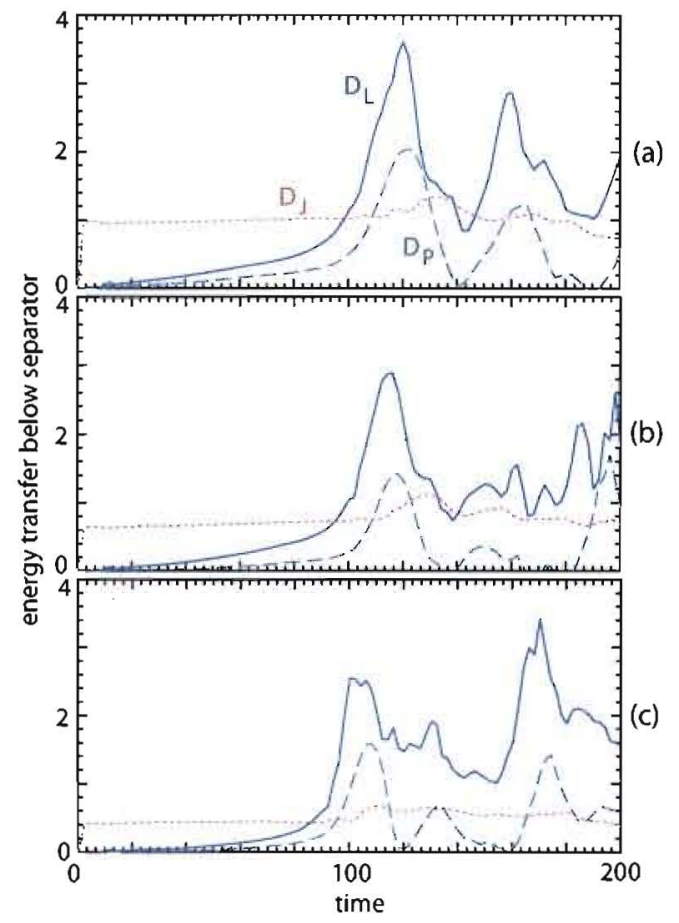

Fig. 11.- Transfer of energy in the downflow region below the reconnection site, integrated over a box $4.2<z<8.2,|x|<2.1$, and $|y|<y_{\max }=40$; (a) corresponds to the case previously discussed with $\eta_{1}=0.005$ and $L_{x}=2.0$, (b) represents a case with the same peak value $\eta_{1}$ but reduced width $L_{x}=0.5$, and (c) represents a case with reduced resistivity $\eta_{1}=0.002$ and $L_{x}=2.0$. Blue lines represent the transfer from magnetic to bulk kinetic energy by the acceleration through the Lorentz force, $D_{L}$, green lines show the transfer from bulk kinetic energy to thermal energy by the work done against the pressure gradient, $D_{P}$, and red lines indicate the direct transfer from magnetic to thermal energy by Joule (or Ohmic) heating.

where $d V=d x d y d z$. Figure 11 shows these quantities for the force-free initial case as functions of time, using three different resistivity models: (a) corresponds to the case previously discussed with $\eta_{1}=0.005$ and $L_{x}=2.0$, (b) represents a case with the same peak value $\eta_{1}$ but reduced width $L_{x}=0.5$, and (c) represents a case with reduced resistivity $\eta_{1}=0.002$ and $L_{x}=2.0$. Blue solid lines represent the transfer from magnetic to bulk kinetic energy by the acceleration through the Lorentz force, $D_{L}$, defined by (11). Green dashed lines show the transfer from bulk kinetic energy to thermal energy by the work done against the pressure gradient, $D_{P}$, defined by (12), and red lines indicate the direct transfer from magnetic to thermal energy by Joule (or Ohmic) heating, $D_{J}$, defined by (10).

Figure 11 shows that Ohmic heating, $D_{J}$, is already present at early times prior to the 
start of reconnection and the fast energy release. This is due to resistive diffusion caused by our model of relatively widely spread resistivity. The effect becomes smaller when the width of the resistive region, $L_{x}$, (Fig. 11b) or the peak resistivity, $\eta_{1}$, are reduced (Fig. 11c). In contrast, the increase of Ohmic dissipation at the time of the fast energy release is small. This confirms the minor role of Ohmic heating in the energy release and transfer process. Fig. 11 further demonstrates that the dominant transfer term is $D_{L}$, representing the acceleration by Lorentz forces. However, due to an approximate balance between the Lorentz forces (11) and pressure gradient forces (12), most of this energy becomes transferred immediately to thermal energy, while only a smaller part remains as bulk kinetic energy. (This is different from the upward open outflow region, where bulk kinetic energy flux is more important.) Consequently, the adiabatic transfer process dominates over direct Joule heating (10) and is primarily responsible for the increase in enthalpy flux.

The effects of the energy transport closer to the chromospheric boundary are demonstrated by Figure 12, which shows the evolution of temperature or average energy (gray scale) and Poynting flux (red contours) at the surface $\mathrm{z}=1$ for the initially force-free case. (Because of our simple reflecting boundary condition at $z=0$, the fluxes vanish directly at the boundary.) The dashed lines represent the separatrices connecting to the reconnection site. Using our units in (1), the maximum temperature or energy in Figure 12 corresponds to $\sim 9 \mathrm{keV}$ (sum of ion and electron energy). The left panels show the temperature evolution on a larger temporal scale, while the right panels show the superposed evolution of Poynting flux around the time of its peak. The peak values correspond to $\sim 0.8 \times 10^{8} \mathrm{~W} / \mathrm{m}^{2}$.

The separatrices and the bands of enhanced temperature in Figure 12 move apart in the $x$ direction, as expected from the progress of reconnection. In addition, the separation of the bands of enhanced temperature in $y$ decreases. The locations of high Poynting flux, which we might tentatively associate with hard X-ray footpoint locations, also move apart, roughly antiparallel to the magnetic neutral line - a behavior which has been observed in a large fraction of solar flares (e.g., Bogachev et al. 2005). Both occur as a consequence of a reduction of shear.

\section{Summary and Conclusions}

We have used resistive MHD simulations to investigate the energy flow and conversion in a three-dimensional extension of the standard flare reconnection scenario, as it is believed to arise in association with the departure of a CME (e.g., Forbes and Acton 1996). Since it is not clear whether the current sheet in the stretched field configuration below the departing $\mathrm{CME}$ is force-free, we have investigated both force-free and non-force-free configurations. The 

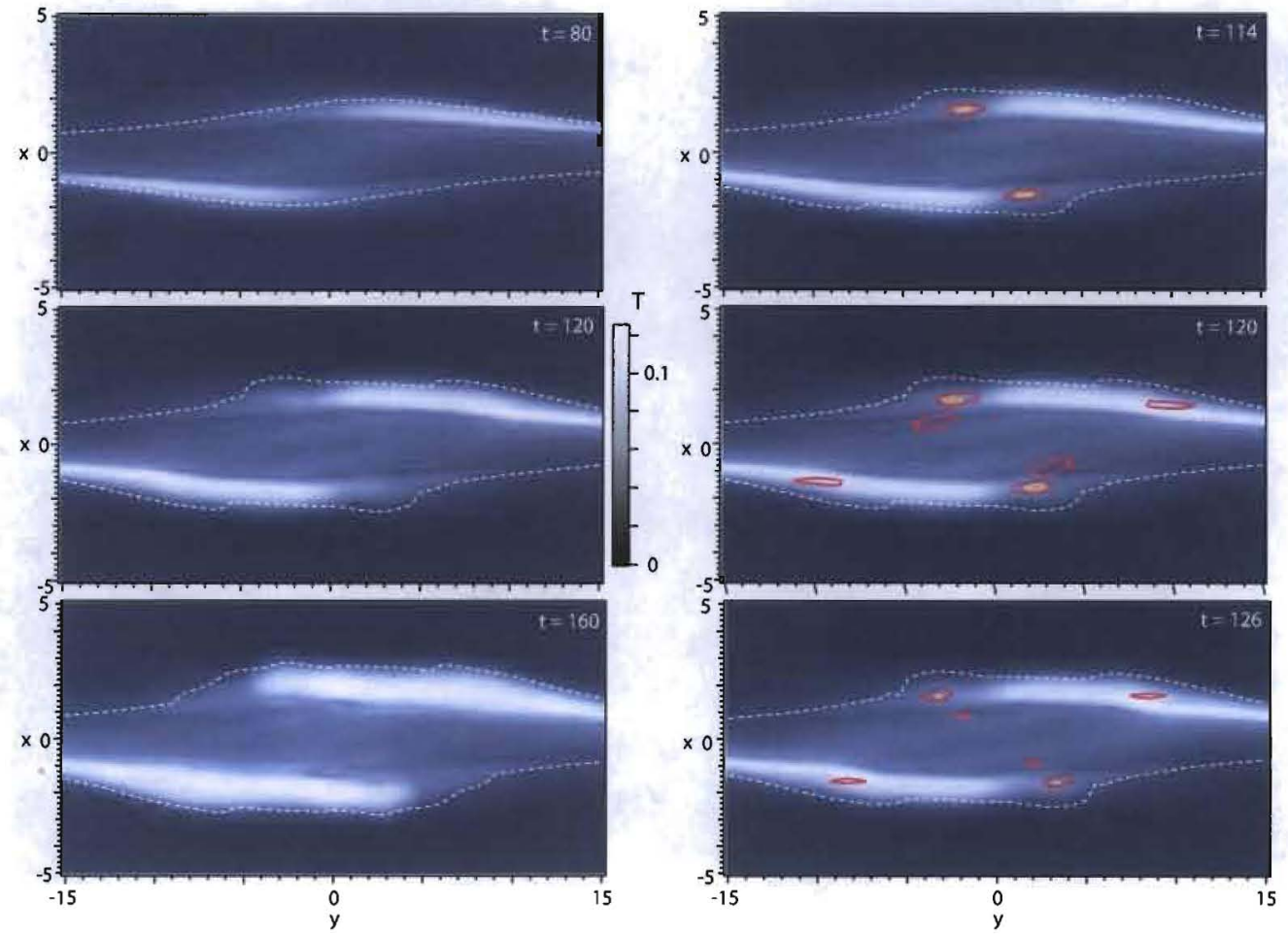

Fig. 12.- Evolution of temperature (gray scale) and Poynting flux (red contours) at a surface $z=1$ for the initially force-free case. The dashed lines represent the separatrices connecting to the reconnection site.

latter require a finite plasma pressure, and accordingly high plasma $\beta$, within the current sheet, whereas the force-free case is characterized by a strong magnetic field component (shear field or guide field) in the direction of the peak current and low $\beta$ throughout. In either case, we have assumed a low plasma $\beta \leq 0.01$ in the outside region (e.g., Gary 2001).

In both cases we found that the dominant energy transfer downward consists of a redirection of incoming Poynting flux and a conversion to enthalpy flux, that is, convected quasi-thermal energy. Magnetic energy released from the surrounding magnetic fields enters the reconnection site as Poynting flux. This incoming Poynting flux is converted to outgoing Poynting flux, enthalpy flux, and bulk kinetic energy flux. The conversion takes place not just within the diffusion region but along extended layers akin to the slow shocks in Petschek's steady-state reconnection model. In the low- $\beta$ case, the downgoing Poynting flux is the dominant contribution, whereas the enthalpy flux dominates in the high- $\beta$ case. The bulk kinetic energy flux is only a minor contribution in the downward direction. The dominance of the downgoing Poynting flux in the low- $\beta$ case is consistent with an alternative to the thick target electron beam model for solar flare energy transport, suggested recently 
by Fletcher and Hudson (2008).

Compressional effects, resulting in gas heating, play a significant role even in low- $\beta$, initially force-free, configurations. This was found already in 2D MHD simulations of reconnection in initially 1D force-free current sheets (Yokoyama and Shibata 2001). The heating near the reconnection site is related to the spatial variation of the guide field. In a force-free current sheet, the guide field maximizes in the center of the current sheet and decreases outward. Consequently, as reconnection proceeds, the guide field that is transported inward toward the reconnection site decreases in time and the plasma pressure at the reconnection site must increase to maintain approximate pressure balance with the exterior magnetic pressure. This heating may result from both Ohmic dissipation and compression.

A more significant heating, however, occurs outside the diffusion region. It is most easily understood as a two-step process (although the two steps occur essentially simultaneously). The first step is the acceleration of the plasma by Lorentz forces in layers akin to Petscheks slow shocks, involving the conversion of magnetic energy to bulk kinetic energy. However, due to pressure gradient forces that oppose the Lorentz forces in approximate, or partial, force balance, the accelerated plasma becomes slowed down and compressed, whereby the bulk kinetic energy is converted to heat, which is mostly transported away by enthalpy flux but may also be deposited by compressional heating. This latter mechanism is obviously not part of the (incompressible) Petschek model. By lowering the background plasma $\beta$ from 0.01 to 0.002 we documented that this heating mechanism persists even for very low $\beta$. It is obvious that the force balance argument is more relevant in the downflow region, whereas it is less important in the outflow above the reconnection site, so that more energy remains in the form of bulk flow in that region. Further pressure and temperature increase then results from the compression of downward collapsing reconnected flux tubes. This pressure increase tends to start near the top of the loops and then propagates downward along the field lines. The propagation speed is essentially the sound speed, which is initially low but increases as the pressure increases.

The pressure increase results in heated loops of about $4 \mathrm{keV}$ electron temperature (assuming equal ion and electron temperatures), which we might associate with soft X-ray flare loops. While this temperature seems to be in the upper range of, or even above, those typically observed (e.g. Feldman 1996; Holman et al. 2003), we note that our approach did not include major loss mechanisms, such as radiation, collisions, and transport through the lower boundary toward the chromosphere and photosphere. The foot points of the high-pressure, high-temperature, field-lines lie on ribbons just inside the magnetic separatrices that connect to the reconnection site. This effect is consistent with the common view of how the bright ribbons in a two-ribbon flare are produced. The characteristic time scale for this pressure 
increase is about $1-2 \mathrm{~min}$.

In addition to the more gradual compressional heating, we also found major energy release and conversion arising from an impulsive increase of the rate of reconnection on a time scale of just a few seconds, which may consist of several bursts. These bursts are related to the break-up of an initially extended, quasi two-dimensional, reconnection line (separator) into smaller, spatially more localized, reconnection sites. Therefore, at particular spots in the lower plane the Poynting flux (and the enthalpy flux in the high- $\beta$ case) become particularly strong - we might tentatively associate these with locations where one might find the brightest flare hard X-ray or white light sources within more extended $\mathrm{H}_{\alpha}$ or ultraviolet/extreme ultraviolet ribbons, such as is commonly observed (e.g., Asai et al. 2002, 2004; Krucker et al. 2005).

In contrast to the low- $\beta$ case, in the high- $\beta$ current sheet, enhanced pressure already exists prior to reconnection and extends also along field lines downward. Accordingly this model produces ribbons of enhanced pressure even prior to reconnection. This makes it less plausible as flare model.

For plausible characteristic parameters of the reconnecting field configuration, we obtain energy release time scales of tens of seconds and downward energy fluxes of the order of $10^{24} \mathrm{~W}=10^{31} \mathrm{erg} / \mathrm{s}$, consistent with estimates inferred from observations for the impulsive phase of flares. Even in the initially force-free, low- $\beta$ case the enthalpy flux may be as large as about $20 \%$ of the Poynting flux.

Our investigations were based on one-fluid MHD simulations. Obviously, the one-fluid approximation does not permit a distinction between thermal and nonthermal populations or between ion and electron energization. The heating observed in the MHD simulations corresponds to an increase in average particle energy in the plasma rest frame, which need not correspond to a Maxwellian distribution. In order to identify to what extent the energy partitioning found in the MHD simulations can be validated by particle approaches, we have also performed particle-in-cell (PIC) simulations, albeit of more localized reconnection problems. Details of these simulations will be reported in a future paper. Here we just note that they confirm our basic MHD results that only a minor amount of the energy released goes into bulk kinetic energy in a closed system or Joule dissipation $\mathbf{j} \cdot \mathbf{E}^{\prime}$, where $\mathbf{E}^{\prime}$ is the electric field in the plasma rest frame. As in the MHD simulations, the dominant outflows from the reconnection site consist of (redirected) Poynting flux and enthalpy flux.

This work was conducted under the auspices of the US Department of Energy, supported by NASA through its Heliophysic Theory, Living With a Star, and Supporting Research and Technology programs. L.F. would like to acknowledge support by the UK STFC under 
rolling grant ST/F002637 and by the European Commission through the SOLAIRE Network (MTRN-CT-2006-035484).

\section{REFERENCES}

Asai A, Masuda S, Yokoyama T, Shimojo M, Isobe H, Kurokawa H and Shibata K 2002 ApJ 578, L91-L94.

Asai A, Yokoyama T, Shimojo M, Masuda S, Kurokawa H and Shibata K 2004 ApJ 611, 557567.

Birn J, Forbes T G and Hesse M 2006 Astrophys. J. 645, 732-741.

Birn J, Forbes T G and Schindler K 2003 Astrophys. J. 588, 578.

Birn J, Galsgaard K, Hesse M, Hoshino M, Huba J, Lapenta G, Pritchett P L, Schindler K, Yin L, Büchner J, Neukirch T and Priest E R 2005 Geophys. Res. Lett. 32, L06105, doi:10.1029/2004GL022058.

Birn J, Gosling J T, Hesse M, Forbes T G and Priest E R 2000 Astrophys. J. 541, 1078-1095.

Birn J, Iinoya F, Brackbill J U and Hesse M 1996 Geophys. Res. Lett. 23, 323.

Bogachev S A, Somov B V, Kosugi T and Sakao T 2005 ApJ 630, 561-572.

Book D L, Boris J P and Hain K 1975 J. Comp. Phys. 18, 248.

Carmichael H 1964 in W. N Hess, ed., 'AAS/NASA Symposium on the Physics of Solar Flares' NASA Washington, DC p. 451.

Emslie A G, Dennis B R, Holman G D and Hudson H S 2005 Journal of Geophysical Research (Space Physics) 110(A9), 11103.

Emslie A G and Sturrock P A 1982 Sol. Phys. 80, 99-112.

Fan Y and Gibson S E 2007 ApJ 668, 1232-1245.

Feldman U 1996 Phys. Plasmas 3, 3203.

Feldman U, Doschek G A, Behring W E and Phillips K J H 1996 ApJ 460, 1034-+.

Feldman U, Doschek G A, Mariska J T and Brown C M 1995 ApJ 450, 441-+.

Fletcher L and Hudson H S 2008 ApJ 675, 1645-1655. 
Forbes T G 2000 Phil. Trans. R. Soc. 358, 711-27.

Forbes T G and Acton L W 1996 ApJ 459, 330.

Forbes T G, Malherbe J and Priest E R 1989 Solar Phys. 120, 285.

Forbes T G and Priest E R 1983 Solar Phys. 84, 169.

Forbes T G and Priest E R 1995 Astrophys. J. 446, 377-389.

Gary G A 2001 Solar Phys. 203, 71.

Hesse M and Birn J 1993 J. Geophys Res. 98, 3973.

Hirayama T 1974 Solar Phys. 34, 323-338.

Holman G D, Sui L, Schwartz R A and Emslie A G 2003 ApJ 595, L97-L101.

Inhester B, Birn J and Hesse M 1992 Solar Phys. 138, 257-281.

Kopp R A and Pneuman G W 1976 Sol. Phys. 50, 85-98.

Krucker S, Fivian M D and Lin R P 2005 Advances in Space Research 35, 1707-1711.

Kuperus M 1976 Solar Phys. 47, 361.

Kusano K 2002 ApJ 571, 532.

Liu W, Liu S, Jiang Y W and Petrosian V 2006 ApJ 649, 1124-1139.

Longcope D W and Priest E R 2007 Physics of Plasmas 14(12), 122905.

Manchester, IV W, Gombosi T, DeZeeuw D and Fan Y 2004 ApJ 610, 588-596.

Melrose D B 1992 ApJ 387, 403-413.

Miller J A, Cargill P J, Emslie A G, Holman G D, Dennis B R, LaRosa T N, Winglee R M, Benka S G and Tsuneta S 1997 J. Geophys. Res. 102, 14631-14660.

Neukirch T, Giuliani P and Wood P D 2007 in J.Birn and E. R Priest, eds, 'Reconnection of Magnetic Fields: MHD and Collisionless Theory and Observations' Cambridge University Press, Cambridge, England p. 281.

Schrijver C J 2007 ApJ 655, L117-L120. 
Schrijver C J, DeRosa M L, Metcalf T, Barnes G, Lites B, Tarbell T, McTiernan J, Valori G, Wiegelmann T, Wheatland M S, Amari T, Aulanier G, Démoulin P, Fuhrmann M, Kusano K, Régnier S and Thalmann J K 2008 ApJ 675, 1637-1644.

Stasiewicz K and Ekeberg J 2008 ApJ 680, L153-L156.

Sturrock P A 1966 Nature 211, 695.

Török T and Kliem B 2005 ApJ 630, L97-L100.

Török T, Kliem B and Titov V S 2004 Astron. Astrophys. 413, L27-L30.

Ugai M 2007 Phys. Plasmas 14, 102904.

Yokoyama T and Shibata K 2001 ApJ 549, 1160. 